\title{
Congestive Cardiac Failure in Children: An Update on Patho-physiology and Management
}

Rashmi Ranjan Das ${ }^{1 *}$, Shasanka Shekhar Panda ${ }^{2}$, Meely Panda ${ }^{3}$ and Sushree Samiksha Naik ${ }^{4}$

${ }^{1}$ Department of Pediatrics, All India Institute of Medical Sciences, Bhubaneswar, India

${ }^{2}$ Department of Pediatrics Surgery, All India Institute of Medical Sciences, Bhubaneswar, India

${ }^{3}$ Department of Community Medicine, Pt BD Sharma PGIMS, Rohtak, India

${ }^{4}$ Department of Obstetrics and Gynecology, SCB Medical College, Cuttack, India

\begin{abstract}
In contrast to adult heart failures, the pediatric heart failure is diverse if both the etiologies and management point of view are considered. If a child gets hospitalized for heart failure, the costs are more compared to adults because of the frequent need for catheter-based interventions or surgery. The medical care can adversely affect the family structure and function due to affection of the parental economic productivity. Besides this, the morbidity also considerably increases because of an increasing survival secondary to the successful application of medical and surgical therapies. That's why clinicians need an up-to-date knowledge about the pediatric heart failure. The present review describes all the aspects of pediatric heart failure including the recent understandings comprehensively.
\end{abstract}

Keywords: Heart failure; Arrhythmias; Venous congestion; Reninangiotensin system

\section{Introduction}

\section{Definition}

When the heart is no longer able to meet the metabolic demands of the body at physiological level, congestive cardiac failure (CCF) occurs [1].

\section{Burden of disease}

Heart failure (HF) is a growing problem worldwide: more than 20 million people around the world are affected and more than 5 million in the United States [2]. The prevalence of HF follows an exponential pattern, and it rises with age. Heart failure affects $2 \%$ of all pediatric inpatient admissions [3].

\section{Etiology and mechanism}

Systolic dysfunction: There is decreased ventricular contractility and failure to increase the stroke volume by the heart to meet the physiologic body demands. Besides this, various factors including the anatomical one (e.g., aortic coarctation) that increases the after-load and neurohumeral factors that increase systemic vascular resistance, leads to systolic dysfunction $[1,2]$.

Diastolic dysfunction: There is decreased ventricular compliance that leads to an increase in the venous pressure (as a compensatory mechanism) to maintain ventricular filling. Causes are: anatomic obstruction preventing ventricular filling (e.g., valvular stenosis), primary reduction in ventricular compliance (e.g., cardiomyopathy), extrinsic factors (e.g., pericarditis and effusion) [1,2].

During acute phase of CCF, the neuro-humeral factors (e.g., sympathetic nervous system and renin-angiotensin system) cause an increase in the myocardial contractility, peripheral vasoconstriction, salt and fluid retention to maintain blood flow and pressure to the vital organs. However, in the chronic CCF phase, continued activity of these same mechanisms at supra-physiological level cause adverse effects (increases in the myocardial oxygen demands, hypertrophy of cardiac myocytes and remodeling of the heart/blood vessels) [1-3].

\section{Signs and symptoms}

The clinical features of heart failure include: increase heart rate (tachycardia), features of venous congestion (right-sided failure causes hepatomegaly, ascites, pleural effusion, edema, increase and jugular venous pressure; left-sided failure causes tachypnea, dyspnea, respiratory distress, and pulmonary edema), and features of low cardiac output (feeding difficulty, lethargy, pallor, sweating, cool periphery, growth failure, and syncope) [3-5].

\section{Differential diagnosis and causes}

Many classes of disorders can result in increased cardiac demand or impaired cardiac function [3-5].

Cardiac causes: arrhythmias (tachyarrhythmias or bradyarrhythmias), structural and functional heart disease (systolic or diastolic dysfunction).

Non-cardiac causes: increase in the preload (e.g., hypervolemia or fluid overload), increase the afterload (systemic hypertension, vasculitis), reduction of oxygen-carrying capacity of the blood (e.g., severe anemia, sepsis), or increase demand (e.g., severe anemia, sepsis) [3].

Neonates and young infants: structural heart diseases are more likely to present during the neonatal period or in young infants $(<2$ months age) with CCF particularly in cases of duct-dependent lesions. In this age group, urgent cardiac evaluation (echocardiography) and start of medication to maintain ductal patency (e.g., prostaglandin E1)

*Corresponding author: Rashmi Ranjan Das, Assistant Professor, Department of Pediatrics, AlIMS, Bhubaneswar - 751019, India, Tel: 91-9438884188; E-mail: dr_rashmipgi@yahoo.com

Received: August 13, 2014; Accepted: October 10, 2014; Published: October 10, 2014

Citation: Das RR, Panda SS, Panda M, Naik SS (2014) Congestive Cardiac Failure in Children: An Update on Patho-physiology and Management. Cardiol Pharmacol 3: 122. doi:10.4172/2329-6607.1000122

Copyright: (c) 2014 Das RR et al. This is an open-access article distributed under the terms of the Creative Commons Attribution License, which permits unrestricted use, distribution, and reproduction in any medium, provided the original author and source are credited. 
is mandatory. If structural heart disease has been ruled out, genetic diseases causing primary cardiomyopathy (e.g., fatty acid oxidation defect) or any other inborn errors of metabolism causing damage to the heart must be investigated. Besides this, respiratory ailments, anemia, and sepsis must be considered and appropriately investigated and managed $[4,5]$.

Infants and older children: in this age group, CCF may be caused by left-sided obstructive disease (e.g., aortic stenosis or coarctation aorta), myocardial dysfunction (myocarditis or cardiomyopathy), hypertension, renal failure or rarely, arrhythmias [5].

The causes can be broadly grouped under following conditions $[4,5]$ :

(i) Cardiac rhythm disturbances caused by: complete heart block, supraventricular tachycardia (PSVT), ventricular tachycardia (VT), and sinus node dysfunction

(ii) Volume overload caused by: structural heart disease (e.g. VSD, PDA, valvular regurgitation, complex cardiac lesions), anemia, and sepsis

(iii) Pressure overload caused by: structural heart disease (e.g., valvular stenosis, coarctation aorta), and hypertension

(iv) Systolic dysfunction caused by: myocarditis, cardiomyopathy (dilated), severe malnutrition, and ischemia

(v) Diastolic dysfunction caused by: cardiomyopathy (hypertrophic, restrictive), and pericardial effusion or cardiac tamponade

\section{Work up}

History taking and physical examination including an assessment of the four-limb blood pressures are crucial in the evaluation of infants or children CCF [3-5].

Whenever is required, appropriatelaboratory testing should include some or all of the following: SPO2 (oxygen saturation), Hemogram including blood counts, serum electrolytes (including renal and liver function tests), and serum calcium. Brain natriuretic peptide (BNP) or $N$-terminal prohormone BNP (NT-proBNP) levels are elevated if there is ventricular dysfunction or dilation [6]. Increased serum BNP level is particularly useful in distinguishing patients with respiratory distress due to CCF from those due to a primary respiratory process. A BNP levels of $>100 \mathrm{pg} / \mathrm{mL}$ is associated with CCF in children [6].

There may be dilutional hyponatremia in the patient with CCF secondary to water retention. Hyperkalemia may represent renal compromise or tissue destruction due to a low cardiac output state. Continue tissue hypoxia may result in lactic acidosis and deplete the serum bicarbonate level. In chronic failure states of longer duration, there may be azotemia or uremia because of reduced renal blood flow [3-5].

In the presence of CCF, there is cardiomegaly observed on the chest radiograph. As with BNP elevation, cardiomegaly may help distinguish patients with CCF from those with respiratory disease (exceptions are restrictive cardiomyopathy, total anomalous pulmonary venous return, and diastolic dysfunction due to mechanical ventilation) [6]. Pulmonary plethora may be present along with pulmonary edema or venous congestion noted on the chest radiograph.

A 12-lead ECG may reveal evidence of structural or coronary artery disease or a complete atrioventricular block or arrhythmia.
Echocardiography is indicated in any child with unexplained CCF to assess cardiac function and identify potential cardiovascular causes [5]. On the other hand, CCF itself is not an echocardiographic diagnosis. Therefore, the underlying etiology is best identified by means of detailed history taking and physical examination, and often by chest radiography.

Pulse oximetry, and a hyperoxia test in newborns, may be useful particularly if cyanotic heart disease causing CCF is suspected [5]. Hyperoxia test may help in distinguishing intracardiac mixing malformations from pulmonary disease in the setting of hypoxia in settings where echocardiography is not easily available. Arterial blood gas (ABG) may show respiratory alkalosis in mild forms of CCF or metabolic acidosis in patients with advanced CCF or a duct-dependent cardiac lesion.

\section{Management}

The management of CCF is difficult and sometimes dangerous without knowledge of the underlying cause. The goals of medical therapy include reducing the preload, enhancing cardiac contractility, reducing the afterload, improving oxygen delivery, and enhancing nutrition. As previously emphasized, the causes of CCF widely vary in children of different age groups. So, the medical management should be tailored to be case specific [3-5].

Preload reduction can be achieved with oral or intravenous (iv) diuretics (eg, furosemide, thiazides, metolazone). Venous dilators (eg, nitroglycerin) can be administered, particularly secondary to severe hypertension in cases with renal failure. Contractility can be supported with iv inotropes (eg, dopamine, dobutamins) or inodilators (eg, dobutamine, inamrinone, milrinone). Digoxin appears to have some benefit in CCF, but the exact mechanism is unclear. Afterload reduction is obtained oral ACE inhibitors or IV agents such as sodium nitroprusside [5].

\section{Acute CCF}

With acute CCF, admission to the ICU for diuresis with iv furosemide and dopamine $(5-10 \mathrm{mcg} / \mathrm{kg} / \mathrm{min})$ or milrinone $(0.3-1.0$ $\mathrm{mcg} / \mathrm{kg} / \mathrm{min}$ ) infusion are appropriate until stabilization is achieved $[7,8]$. Placement of a central venous or pulmonary artery catheter to monitor venous pressure and cardiac output is very useful and should be tried in each case.

Nitrates (nitroprusside, nitroglycerin) or nesiritide may be useful in patients with elevated pulmonary capillary wedge pressure and pulmonary congestion due to their venodilator effects. Nesiritide has an additional benefit of reversing deleterious neurohumoral responses and increasing natriuresis $[5,9]$.

\section{Chronic or stable CCF}

They can be managed at the outpatient setting by initiating several agents $[8,10]$. Afterload reduction using an ACE-inhibitor is indicated in the presence of left ventricular dysfunction. In mild forms of CCF, low-dose furosemide $(1 \mathrm{mg} / \mathrm{kg} /$ dose per-oral twice daily) may be initiated with addition of another agent having inotropic effect (e.g., digoxin) or reducing the afterload (e.g., ACE inhibitor).

For more severe CCF, diuretic therapy with oral furosemide may be increased to $2 \mathrm{mg} / \mathrm{kg} /$ dose thrice daily or a second agent (e.g., hydrochlorothiazide or metolazone) may be added. To be most effective, hydrochlorothiazide and metolazone are best administered simultaneously with furosemide to achieve their synergistic effect [5]. 
Afterload reduction is indicated in patients who have large left-toright shunts (e.g., ventricular septal defect or patent ductus arteriosus), left-sided valvular regurgitant lesions (e.g., aortic insufficiency or mitral regurgitation), or poor systolic function (e.g., myocarditis or dilated cardiomyopathy). ACE-inhibitors are the medications for all these conditions. Alternatively, an angiotensin receptor blocker (ARB), such as losartan may be used in patients in whom ACE-inhibitor related side effects (e.g., cough) may be unacceptable [11].

For patients taking oral furosemide for a longer period (without ACE-inhibitors), spironolactone should be added for its potassiumsparing effect and theoretical beneficial effects on cardiac remodeling [12]. Alternatively, serum potassium levels may be monitored, and appropriate supplementation should be provided.

Hyponatremia often accompanies congestive heart failure and is caused by water retention in response to the anti-diuretic hormone. It may be exacerbated by the use of diuretics. However, sodium supplementation is almost never indicated in infants or children with CCF except in emergency situations (e.g., development of seizure or encephalopathy) [5]. Severe hyponatremia is generally best managed by reducing the dose of diuretics or by restricting fluid intake, although the latter has little use in small children. In children on prolonged diuretics, serum electrolyte levels should be checked every few months or so.

There has been a recent trend towards the use of $\beta$-blockers in pediatric patients with CCF $[13,14]$. Beta-blockers are an essential part of standard therapy in adults with CCF and are therefore also expected to be beneficial in children. The Cochrane review included 3 studies (203 participants) [15]. Out of the 3 studies, 2 small studies (20 and 22 children respectively) showed an improvement of CCF, while the larger study (161 participants) showed no evidence of benefit over placebo in the composite outcomes $(p=0.74)$. However, the study populations showed vast differences with regard to treatment (choice of beta-blocker, dosing, duration of treatment), age and age range of the participants and in particular with regard to condition (etiology and severity of heart failure; homogeneity of condition in the study population). The review concluded that, there are not enough data to recommend or discourage the use of betablockers in children with congestive heart failure. Another recently conducted systematic review examined the impact of carvedilol and conventional therapy on the clinical outcome in children with chronic heart failure due to impaired systemic ventricle systolic function, and found a beneficial effect [13]. In another prospective study, improvement in ventricular function and clinical symptoms was seen on oral carvedilol added to standard drug therapy in pediatric patients with dilated cardiomyopathy and moderate to severe ventricular dysfunction [16].

The role of the oxygen-carrying capacity of the blood is often overlooked in the management of CCF. Anemia aggravates heart failure by increasing the demands for cardiac output. So, careful attention to iron stores or the administration of packed red cell transfusions may result in significant improvement in the symptoms of CCF [5].

Besides aggressive medical management, nutrition is crucial in the management of chronic CCF $[4,5]$. Inadequate weight gain may delay the surgery unnecessarily as poor weight during pre-operative period has been shown to increase the hospital stay as well as the mortality. In infants and young children, CCF increases the metabolic demands while making feeding itself more difficult. Making the feeds calorie dense or in some cases, nasogastric or gastrostomy feedings is necessary to maintain the child's growth. As such, the success of medical therapy is judged as per the child's growth. A failure to thrive (FTT) is an indication for increased medical management or, when the option is available, surgical repair of underlying condition [5].

\section{Device based therapies}

Cardiac resynchronization therapy (CRT) is an emerging therapy in the treatment of chronic CCF [17]. CRT involves the use of biventricular pacemakers to improve ventricular function by electrically adjusting the timing of right and left ventricular contraction to optimize wall motion and increase filling time. It increases stroke volume as well as reverses the remodeling or delay the disease progression. In pediatric patients, the role of CRT is limited and it is also more difficult to place inside a child's heart.

Mechanical support may be used in the treatment of acute CCF (i.e., myocarditis or post-cardiac surgery cases) or in chronic uncontrolled CCF as a bridge to recovery or cardiac transplant $[18,19]$. Extracorporeal membrane oxygenation (ECMO) therapy may be quickly initiated in unstable patients. Generally, its duration of use is limited to days to weeks because complications related to infection or anticoagulation.

Ventricular assist devices (eg, Berlin Heart or Thoratec VAD) may be used to provide long-term ventricular support [20]. Currently, none are approved for permanent therapy in pediatric patients and should only be used as a bridge to transplantation in appropriate candidates [21].

\section{Others}

Other treatment modalities tried are: immunosuppressants (e.g. heart failure in myocarditis); antibody therapy (iv immunoglobulin for CCF secondary to myocarditis); arginine vasopressin (post-operative myocardial dysfunction) $[5,18,22,23]$.

\section{References}

1. Ramakrishnan S, Kothari SS, Bahl VK (2005) Heart Failure-Definition and Diagnosis. Indian Heart J 57:13-20.

2. Fang J, Mensah GA, Croft JB, Keenan NL (2008) Heart failure-related hospitalization in the U.S., 1979 to 2004. J Am Coll Cardiol 52:428-434.

3. Sommers C, Nagel BH, Neudorf U, Schmaltz AA (2005) Congestive heart failure in childhood. An epidemiologic study. Herz 30:652-662.

4. Kay JD, Colan SD, Graham TP (2001) Congestive heart failure in pediatric patients. Am Heart J 42:923-928

5. Chaturvedi V, Saxena A (2009) Heart failure in children: clinical aspect and management. Indian J Pediatr 76:195-205.

6. Law YM, Keller BB, Feingold BM, Boyle GJ (2005) Usefulness of plasma B-type natriuretic peptide to identify ventricular dysfunction in pediatric and adult patients with congenital heart disease. Am J Cardiol 95:474-478

7. Mehra MR (2006) Optimizing outcomes in the patient with acute decompensated heart failure. Am Heart J 151:571-579.

8. Working Group on Management of Congenital Heart Diseases in India, Saxena A, Juneja R, Ramakrishnan S (2009) Drug therapy of cardiac diseases in children. Indian Pediatr 46:310-338.

9. Jefferies JL, Price JF, Denfield SW, Chang AC, Dreyer WJ, et al. (2007) Safety and efficacy of nesiritide in pediatric heart failure. J Card Fail 13:541-548.

10. Rosenthal D, Chrisant MR, Edens E, Mahony L, Canter C, et al. (2004) International Society for Heart and Lung Transplantation: Practice guidelines for management of heart failure in children. J Heart Lung Transplant 23:1313-1333

11. Konstam MA, Neaton JD, Poole-Wilson PA, Pitt B, Segal R, et al (2005) Comparison of losartan and captopril on heart failure-related outcomes and symptoms from the losartan heart failure survival study (ELITE II). Am Heart J 150:123-131. 
Citation: Das RR, Panda SS, Panda M, Naik SS (2014) Congestive Cardiac Failure in Children: An Update on Patho-physiology and Management. Cardiol Pharmacol 3: 122. doi:10.4172/2329-6607.1000122

Page 4 of 4

12. Pitt B, Zannad F, Remme WJ, Cody R, Castaigne A, et al. (1999) The effect of spironolactone on morbidity and mortality in patients with severe heart failure. Randomized Aldactone Evaluation Study Investigators. N Engl J Med 341:709717.

13. Prijic S, Kosutic J, Vukomanovic V, Prijic A, Bjelakovic B, et al. (2014) BetaBlockers (Carvedilol) in Children with Systemic Ventricle Systolic Dysfunction - Systematic Review and Meta-Analysis. Rev Recent Clin.

14. Buchhorn R, McConnell ME (2014) Beta blockers in childhood heart failure why not? Int J Cardiol 175:211-212.

15. Frobel AK, Hulpke-Wette M, Schmidt KG, Laer S (2009) Beta-blockers for congestive heart failure in children. Cochrane Database Syst Rev 21: CD007037.

16. Saxena A, Anil OM, Juneja R (2013) Clinical and echocardiographic outcome in patients receiving carvedilol for treatment of dilated cardiomyopathy Indian J Pediatr 80: 549-554.

17. Janousek J, Gebauer RA (2008) Cardiac resynchronization therapy in pediatric and congenital heart disease. Pacing Clin Electrophysiol 31: S21-S23.

18. Duncan BW, Bohn DJ, Atz AM, French JW, Laussen PC, et al. (2001) Mechanical circulatory support for the treatment of children with acute
Fulminant myocarditis. J Thorac Cardiovasc Surg 122: 440-448.

19. Stiller B, Dahnert I, Weng YG, Hennig E, Hetzer R, et al. (1999) Children may survive severe myocarditis with prolonged use of biventricular assist devices. Heart 82: $237-240$

20. Shaddy RE (2001) Optimizing treatment for chronic congestive heart failure in children. Crit Care Med 29: S237-S240.

21. Canter CE, Shaddy RE, Bernstein D, Hsu DT, Chrisant MR, et al. (2007) Indications for Heart Transplantation in Pediatric Heart Disease: A Scientific Statement from the American Heart Association Council on Cardiovascular Disease in the Young; the Councils on Clinical Cardiology, Cardiovascular Nursing, and Cardiovascular Surgery and Anesthesia; and the Quality of Care and Outcomes Research Interdisciplinary Working Group. Circulation 115: 658-676.

22. Robinson JL, Hartling L, Crumley E, Vandermeer B, Klassen TP (2005) A systematic review of intravenous gamma globulin for therapy of acute myocarditis. BMC Cardiovasc Disord 5:12.

23. Hia CP, Yip WC, Tai BC, Quek SC (2004) Immunosuppressive therapy in acute myocarditis: an 18 year systematic review. Arch Dis Child 89: 580-584. 